\title{
Clumpy Galaxies in the Early Universe
}

\author{
Debra Meloy Elmegreen \\ Dept. of Physics \& Astronomy, Vassar College, Poughkeepsie, NY 12604 USA \\ email: elmegreen@vassar.edu
}

\begin{abstract}
Clumpy galaxies are prominent in the early Universe. We present morphological and photometric properties of a wide range of galaxy types and their star-forming clumps in the Hubble Ultra Deep Field. Sizes, scale lengths, and scale heights suggest that galaxies grow by a factor of 2 from $z=4$ to the present, and that thick disks are present in the early Universe. The largest clumps of star formation are $10^{7}-10^{9} M_{\odot}$ in different galaxies, much more massive than large star-forming complexes in local galaxies. Dissolved clumps may account for both the exponential disks and the early thick disks of spirals and proto-spiral galaxies.
\end{abstract}

Keywords. galaxies: structure, galaxies: evolution, galaxies: high-redshift

\section{Introduction}

Galaxies in the early Universe are peculiar compared with normal local spiral and elliptical galaxies (Abraham et al. 1996, Conselice et al. 2005, Elmegreen et al. 2005a). Chain galaxies, characterized by linear structures with clumpy substructure, were first noticed in the Hawaiian deep field surveys (Cowie, Hu, \& Songaila 1995). Tadpole galaxies, with a big star-forming clump at one end and a long, sometimes wiggly tail, were seen in the Hubble Deep Fields (van den Bergh 2002). Luminous diffuse objects (LDOs) were noted by Conselice et al. (2004). Some LDOs have disk-like collections of clumps with no central bulge or exponential disk; we refer to this subset as clump clusters (Elmegreen et al. 2005a, Elmegreen \& Elmegreen 2005). Double galaxies have two prominent clumps that may signify mergers. Spirals and ellipticals are also present in deep fields, although with decreasing numbers at redshifts greater than 1.5. A comparison of morphological and photometric properties of galaxies as a function of redshift provides insight into their formation and possible evolution.

\section{Data Reduction and Analysis}

The Hubble Ultra Deep Field (UDF), imaged with the Advanced Camera for Surveys (ACS) in B,V, i, and z filters, contains some 10,000 galaxies out to redshift $\sim 6$ (Beckwith et al. 2006). We classified 1003 galaxies larger than 10 pixels into morphological categories of chain, double, tadpole, clump cluster (these four comprise the clumpy classes; Fig. 1), spiral, and elliptical, based on visual inspection, contour plots, and radial profiles of galaxies in the i filter. Using the Bayesian photometric redshift method (Benitez 2000), we determined their photometric redshifts and spectral types (Elmegreen et al. 2006).

\section{Results}

\subsection{Morphology}

For each galaxy, we evaluated the Sersic index, n, using GALFIT (Peng et al. 2002). This parameter is useful for understanding the overall radial light distribution in a galaxy. 


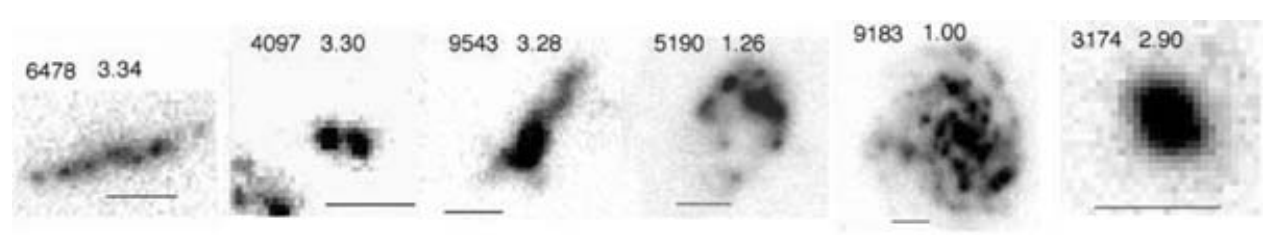

Figure 1. Chain, double, tadpole, clump cluster, spiral, and elliptical galaxies in i-band, with UDF catalog number and redshift indicated. The lines are $1^{\prime \prime}$.

The four clumpy galaxy morphological classes have $n \sim 0-1$ at all redshifts, indicating flat radial light profiles. In contrast, the spirals peak at $n=1$, as expected for normal exponential disks, although they have a range of indices across all redshifts. Many of the elliptical galaxies have fits of $n>2.5$, indicating spheroid-dominated systems, although others show flatter profiles. This wide range of Sersic indices for ellipticals, seen also in local galaxies, was reproduced in simulations of mergers by Aceves et al. (2006).

The axial ratio distributions indicate that chain and clump cluster galaxies are the same type of object; namely, disks viewed edge-on (chains) or more face-on (clump clusters). Simple models of expected axial ratios for a random distribution of clumps in a plane with some thickness fit the observed axial ratio distribution best for a flattening of 3:1, indicating that the disks are thick. The UDF spiral axial ratio distribution similarly indicates thick disks. The axial ratio distribution of ellipticals is the same at high redshift as locally, implying that the ellipticity ranges are the same and that outer parts of ellipticals relaxed quickly (Elmegreen et al. 2005).

\subsection{Clumpy structures and redshift trends}

The number density per co-moving volume was calculated for each morphological type as a function of redshift (Elmegreen et al. 2006). The clumpy galaxy types are the most abundant morphologies at high $z$. This result is consistent with studies of peculiar and normal galaxies in the HDF (Abraham et al. 1996; Ferreras et al. 2005; Conselice 2005), and in the UDF parallel fields (Menanteau et al. 2006; de Mello et al. 2006). At $z<1$, the spirals have about the same number density as the sum of the chains and clump clusters.

The spectral type $t_{b}$ is determined from the observed galaxy colors by comparison with redshifted galaxy templates, with $t_{b}=1$ for elliptical-like (red) colors, 2 for earlytype spirals, 3-4 for late-type, and 5-8 for starbursts. At high $z$, only UV-bright galaxies are seen, so the average $t_{b}$ is 6 for all morphological types. Clumpy types are starburst at low $z$ also, suggesting that they form or light up over a large $z$ range (Elmegreen et al. 2006). Spirals and ellipticals have normal spectral types at $z<1$. Red spirals and ellipticals are present beyond $z \sim 1.5$ in diminished numbers (Labbe et al. 2005; Franceschini et al. 2005), but they are bandshifted out of the ACS filters.

Clump cluster galaxies are nearly pure clump systems, with no bulge and no exponential disk. The clump flux fraction is much higher in clump clusters than in spirals, indicating that the underlying spiral disks are brighter than the underlying disks in clump clusters. Some $30 \%$ of ellipticals are also clumpy (Elmegreen, Elmegreen, \& Ferguson 2005; also in the Tadpole field, Menanteau et al. 2004), although their clump masses are typically $10^{6}-10^{8} M_{\odot}$ rather than the $10^{8}-10^{9} M_{\odot}$ observed in the clump clusters. The ages in all cases are a few $\times 10^{8}$ yr. Clumps may form in situ by gravitational instabilities if gas accretes smoothly to the disks (e.g., Immeli et al. 2004), or may be accreted (Brook et al. 2004); their colors and sizes match those of isolated clumps in the UDF (Elmegreen et al. 2005b). 


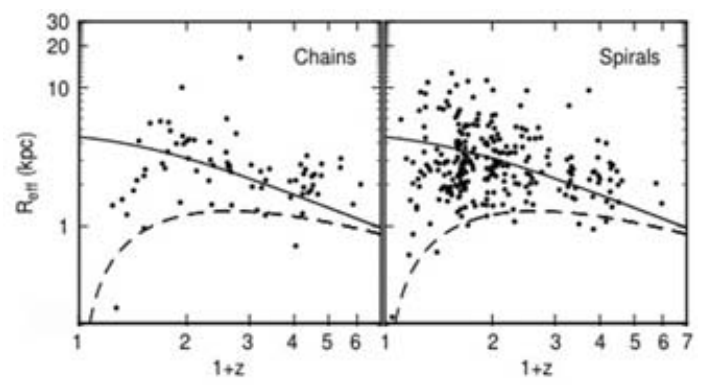

Figure 2. Size as a function of redshift for chain and spiral galaxies in the UDF. Dashed line is the observed limit; solid line is a $10^{10} M_{\odot}$ galaxy (Mo, Mau, \& White 1998).

\subsection{Radial profiles and exponential disk origins}

The radial profiles of spiral galaxies differ from those of chains and clump clusters. Spirals have exponential profiles with color gradients, whereas chain and clump cluster profiles are flat except for clump peaks along the length, and show no color gradients. Also, the average disk surface brightness of clump clusters is about 2 mag fainter than the average for spirals (Elmegreen, Elmegreen, \& Hirst 2004). However, the clump flux density and number density follow the same exponential fall-off for both spirals and clump clusters, which suggests that clumps may disperse (on time scales of a few orbits) to make a smooth disk. Eventually, the clump clusters become spirals (Elmegreen \& Elmegreen 2005; Elmegreen et al. 2005).

The size $r_{\text {eff }}$ of each galaxy, determined from GALFIT, shows that galaxies of all types are about half their current average size at $z \sim 4-5$ (Fig. 2). Furthermore, the spiral scale lengths at high $z$ are about half the local average (Elmegreen et al. 2006). Vertical scale heights of $\sim 1 \mathrm{kpc}$ were derived for disks in both edge-on spirals and chain galaxies, based on sech ${ }^{2}$ fits along with psf deconvolutions of star profiles. The vertical scale height to radial scale length ratio of $\sim 3$ indicates that the disks are thick (Elmegreen \& Elmegreen 2006a). There is no correlation of scale height with redshift, although galaxies are redder with increasing vertical height. Clump diameters are $\sim 80 \%$ of the disk height. The tiny displacement of clump centroids in chain galaxies from the midplane suggests that clumps formed in the disk rather than being accreted. Comparison of colors with population synthesis evolution models indicates that thick disk ages are $\sim 1$ Gyr in the UDF, with continuous or slowly declining star formation.

\subsection{Ring galaxies}

Interactions and mergers are common at all redshifts, and play an important role in the evolution of galaxies (e.g., Conselice 2006). The GEMS (Rix et al. 2004) and GOODSSouth (Giavalisco et al. 2004) surveys, which are centered on the UDF, do not reach as faint a limiting magnitude as the UDF but cover 81 and 18 times the area, respectively, so are useful for examining interactions. Spectrophotometric redshifts have been determined by the COMBO-17 survey (Wolf et al. 2003). Three dozen ring galaxies were observed out to redshift $z=1.4$ in these fields (Elmegreen \& Elmegreen 2006b). Some ring galaxies have close companions and appear to be collisional rings such as those modeled by Struck-Marcell \& Appleton (1987), while other isolated examples may be resonance rings (Buta \& Combes 1996). Some galaxies look like bent chain galaxies, with apparently warped disks from interactions. The star-forming clumps in the chain and bent chain galaxies are bluer than those in the ring galaxies, signifying younger ages (about $10^{7} \mathrm{yr}$ compared with $10^{8} \mathrm{yr}$ for the ring galaxies). Clump masses range from $10^{6}-10^{8} M_{\odot}$. 


\section{Conclusions}

Clumpy galaxy morphologies dominate at high redshift. UDF galaxies are mostly starburst systems; red galaxies cannot be observed in the ACS filters at $z>2$. High $z$ galaxies are dominated by massive $10^{8} M_{\odot}$ clumps, which could be accretions but are more likely in situ star formation. Dissolved clumps may account for both the build-up of an exponential disk and the early thick disk. Galaxies grow in overall size, scale length, and scale height by a factor of $\sim 2$ from $z=5$ to $z=1$. Ring galaxies are common in the GEMS fields at $z=0.5-1.4$; some may be collisional rings formed by interactions, while others may be resonance rings.

\section{Acknowledgements}

I gratefully acknowledge the American Astronomical Society for a travel grant, the International Astronomical Union for registration fees, and Vassar College for further travel support. This research was done in collaboration with Bruce Elmegreen and several undergraduate students.

\section{References}

Abraham, R. et al. 1996, ApJS, 107, 1

Aceves, H., Velazquez, H., \& Cruz, F. 2006, astro-ph/0601412

Beckwith, S. et al. 2006, astro-ph/0607632

Benitez, N. 2000, ApJ, 536, 571

Brook, C. Kawata, D., Gibson, B., \& Freeman, K. 2004, ApJ, 612, 894

Buta, R., \& Combes, F. 1996, Fund. Cosmic Physics, 17, 95

Conselice, C. 2006, ApJ, 638, 686

Conselice, C. 2005, in Proceedings of ESO workshop on Multiwavelength mapping of galaxy formation and evolution, eds. A. Renzini and R. Bender (Berlin: Springer-Verlag), 163

Conselice, C., Blackburne, J., \& Papovich, C. 2005, ApJ, 620, 564

Conselice, C., et al. 2004, ApJ, 600, L139

Cowie, L., Hu, E., \& Songaila, A. 1995, AJ, 110, 1576

de Mello, D., et al. 2006, Wadadekar, Y., Dahlen, T., Casertano, S., \& Gardner, J. 2006, AJ, 131,216

Elmegreen, B., \& Elmegreen, D. 2005, ApJ, 627, 632

Elmegreen, B., \& Elmegreen, D. 2006a, ApJ, 651, in press

Elmegreen, D., \& Elmegreen, B. 2006b, ApJ, 651, in press

Elmegreen, D., Elmegreen, B., \& Ferguson, T. 2005, ApJ, 623, L71

Elmegreen, D., Elmegreen, B., \& Hirst, A. 2004, ApJ, L604, 21

Elmegreen, D., Elmegreen, B., Ravindranath, S., \& Coe, D. 2006, ApJ, submitted

Elmegreen, D., Elmegreen, B., Rubin, D., \& Schaffer, M. 2005a, ApJ, 631, 85

Elmegreen, D., Elmegreen, B., Vollbach, D., Foster, E. \& Ferguson, T. 2005b, ApJ, 634, 101

Ferreras, I., et al. 2005, ApJ, 635, 243

Franceschini, A., et al. 2006, A\&A, 453, 397

Immeli, A., Samland, M., Westera, P., \& Gerhard, P. 2004, ApJ, 611, 20

Giavalisco, M., et al. 2004, ApJ, L600, 93

Labbe, I., et al. 2005, ApJ, 624, L81

Menanteau, F., et al. 2004, ApJ, 612, 202

Menanteau, F., et al. 2006, AJ, 131, 208

Mo, J., Mao, S., \& White, S. 1998, MNRAS, 295, 319

Peng, C.Y., Ho, L., Impey, C., \& Rix, H.-W. 2002, AJ, 124, 266

Rix, H.-W. et al. 2004, ApJS, 152, 163

Struck-Marcell, C., \& Appleton, P. 1987, ApJ, 323, 480

van den Bergh, S. 2002, PASP, 114, 797

Wolf, C., et al. 2003, A\&A, 401, 73 


\section{Discussion}

EDWARD TAYLOR: It's an impressive volume of work, but I am concerned about two things. First, you've made no mention of potential systematics with redshift, including morphological K-corrections and SB effects. Secondly, in what sense is it meaningful to extract Sersic parameters for manifestly non-Sersic objects?

Debra Elmegreen: Yes, good questions. The basic shapes look the same at all observed redshifts (we're primarily seeing star formation regions). Bandshifting effects are taken into account in our population synthesis models. We have considered selection effects due to decreasing surface brightness due to cosmological dimming. Clumpy galaxies still show up, since the clumps are so bright. Spiral galaxies are most affected; we begin to lose them at $z>2$ if they are face-on, as we have modeled. We use Sersic index to get a sense of the radial light distribution, since we want to understand the origin of spiral exponential disks. Edge-on spirals with star-forming regions don't look exponential to the eye, but they are. Chain galaxies, however, are not exponential, even though they resemble edge-on spirals. So the Sersic index is one more piece of information.

JAN PALOUŠ: For clumpy chains and tadpole galaxies, do you have an idea on their 3D-shape?

Debra Elmegreen: Based on axial ratio distribution, and the similarity of chain and clump cluster galaxies, we believe chains are disks, that is clump cluster galaxies seen edge-on. Tadpoles are not so clear, their large end clumps and sometimes wiggly tails suggest they may be mergers. We need kinematic information in order to know. 